\title{
APPLICATION OF TOMOGRAPHY IMAGES TO COMPOSITE SOLID PROPELLENTS
}

\section{LIZANDRO CANALES, JUAN NEYRA, JOSE LUIS, NERY UGARTE, WILVER AUCCAHUASI}

\author{
Comisión Nacional de Investigación y Desarrollo Aeroespacial del Perú, Lima, Perú
}

\begin{abstract}
Quality validation of solid compound propellants is a basic process in the development of the power generator for rocket propulsion. The choice of a specific methodology is based on its effectiveness. The purpose of the study is to support the use of tomographic images as a tool for visualization of the internal part of the solid composite propellant since computed tomography is a non-destructive test method.The results show how faults in the propellant that could generate undesired effects in its combustion are identified through the cuts, as well as verifying the homogeneity of the propellant structure

KEYWORDS: Computed tomography, non-destructive tests, tomographic images, tomography inspection, solid propellant
\end{abstract}

Received: Jun 08, 2020; Accepted: Jun 28, 2020; Published: Sep 25, 2020; Paper Id.: IJMPERDJUN20201418

\section{INTRODUCTION}

The technology of solid propellants applied in the civil and military field in its development stage needs the reliability and security of its operation, for which it must be supported by physical and chemical tests and / or tests, throughout the development stage; The presence of gaps, related to manufacturing imperfection, causes a greater decrease in the propellant density and the generation of a discontinuity in the medium, with the consequent safety problems related to heat transfer and mechanical properties. [1] One of the tests is to be able to visualize the entire internal volume of the propellant without damaging its structure, using a tomograph that provides images with submillimeter spacings along the grain. The images allow the anomalies to be accurately identified in the final stage of the propellant manufacturing process, this is made visible in the images by the change in the color contrasts that represent cavities, fissures, porosities or other imperfections of different magnitudes. It should be mentioned that not all the detected defects will influence the development of the combustion, this will depend on its location, shape and type. [two]. Likewise, it enables us to relate the anomalies detected with the mixing and molding processes to make the necessary corrections, if applicable. This method allows the inspection of the internal configuration of the rocket motor considering its details [3].

\section{METHODS Y MATERIALS}

The term computed tomography (CT) refers to an imaging procedure using X-rays, which are processed by the computer generating cross-sectional images or sections of the propellant. These cuts contain more detailed information than conventional X-rays. This method can be used to determine the relative density of materials, locate inclusions or defects, measure the extent of erosion and ablation in composite materials [4] Once the machine's computer collects several successive cuts, they can be digitally "stacked" to form a three-dimensional image that 
allows to digitally reconstruct the solid propellant at scale, where the internal surface can be observed in each cut. It is analyzed by interpreting the variations in density reflected in the intensity of colors; from white to black in different shades, black represents a cavity or crack within the propellant while the metallic particles are identified with the color white.

This procedure has been developed for polyvinyl chloride (PVC) based solid propellants in order to identify cavities, cracks or other anomalies that can cause an increase in the rate of combustion and lead to malfunctions of the rocket engine. Detection of defects and characterization of the solid rocket propellant were thus possible. [5]

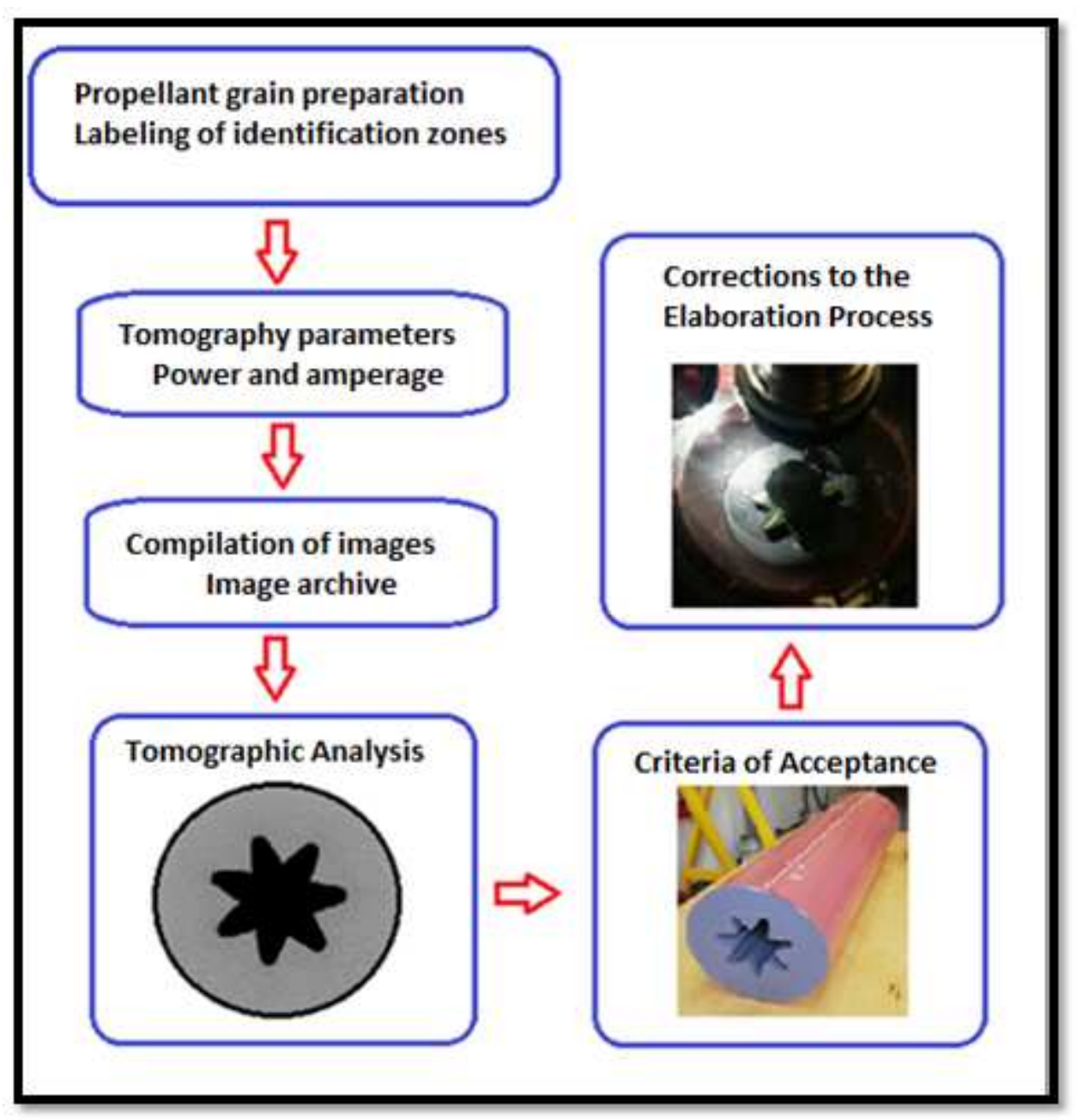

Figure 1 : Tomographic analysis process diagram

\section{PROPELLANT PREPARATION}

The labeling is carried out on the external surface of the propellant, identifying the head area and the nozzle area, establishing an entrance direction to the tomograph to locate all the cuts from the beginning to the end.

\section{TOMOGRAPHY PARAMETERS}

The equipment used was a TEM GE Brightspeed scanner with 16 detector lines, voltage in the X-ray tube of 80 - $140 \mathrm{kV}$, allowing high resolution studies to be carried out; establishing the following parameters for carrying out the analysis. 


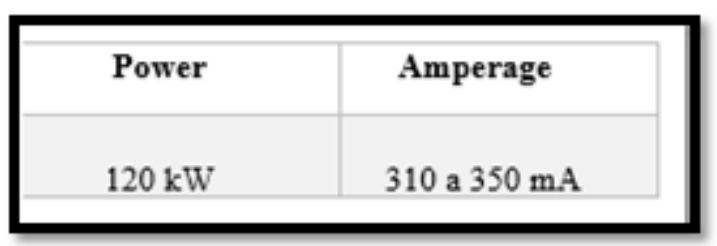

Figure 2 : Tomographic analysis process diagram

\section{MAIN FILTERS USED IN THE ANALYSIS ACCORDING TO THE ESTABLISHED ORDER}

Filter to determine the homogeneity of the propellant charge and bonding of the inhibitor jacket.

\begin{tabular}{|l|l|}
\hline Main Filter & Secondary Filter \\
\hline Window name: Filter 1 & Window name: Filter 2 \\
Window center: 800 & Window center: 1000 \\
Window width : 1800 & Window width: 3500 \\
\hline
\end{tabular}

Figure 3 : Filter types

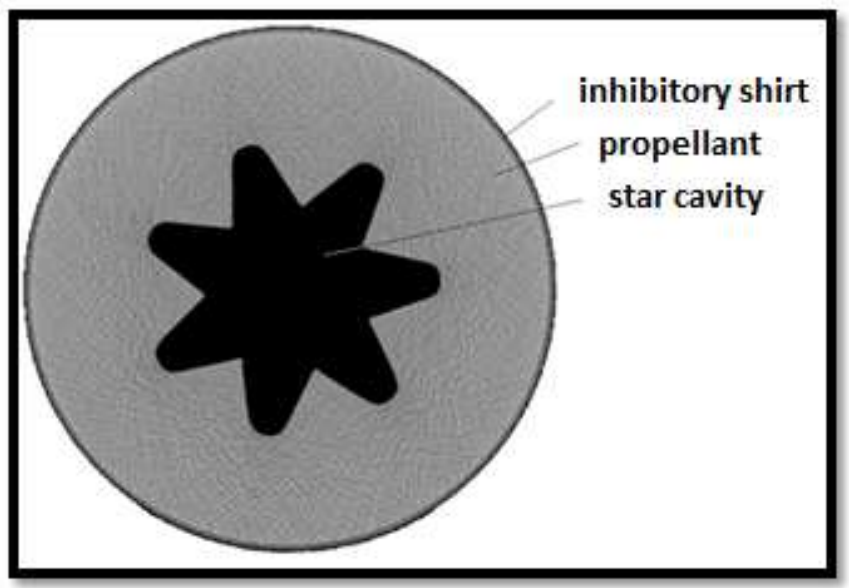

Figure 4 : Radial cut of PVC propellant

Figure 1 shows a section of the PVC propellant, the filter of table 1 allows to visualize the different densities of grain composition such as: propellant (gray color), inhibitory jacket (dark gray) and star core cavity (black color). In the same way in figure 2 we can see elements of higher density of white color that represents agglomerations of aluminum.

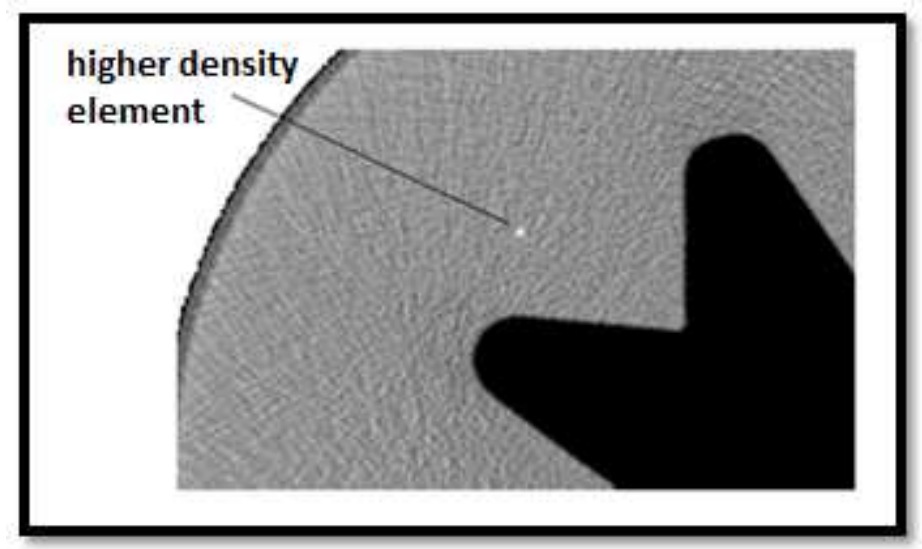

Figure 5 : Filters to determine cavities or elements of higher density in the propellant. 


\begin{tabular}{|l|l|}
\hline \multirow{2}{*}{ Main Filter } & Window name: Filter 3 \\
& Window center: 50 \\
& Window width: 130 \\
\hline \multirow{3}{*}{ Secondary Filter } & Window name: Filter 4 \\
& Window center: 50 \\
& Window width: 80 \\
\hline \multirow{2}{*}{ Secondary Filter } & Window name: Filter 5 \\
& Window center: 50 \\
\hline & Window width: 350 \\
\hline
\end{tabular}

Figure 6 : Filters to determine cavities or elements of higher density in the propellant.

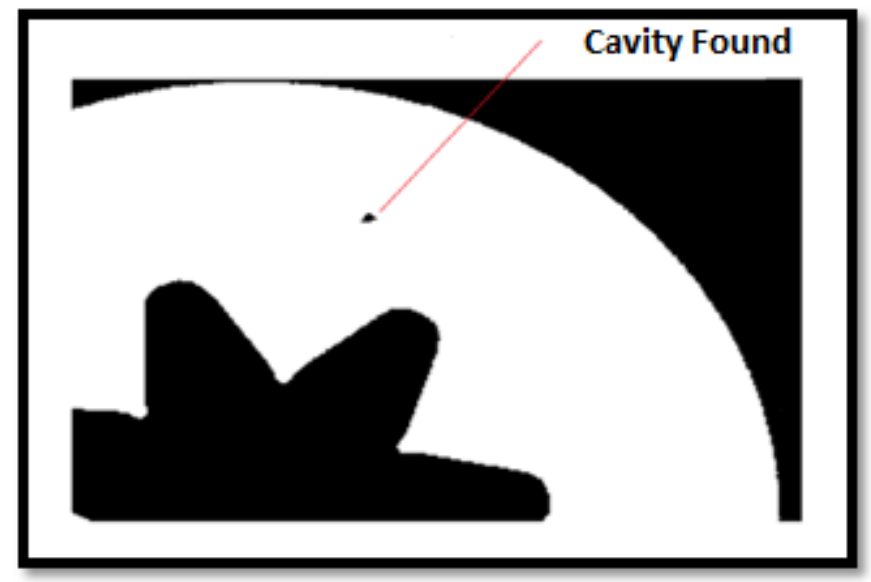

Figure 7 : Identifies areas of low density related to cavity inside the propellant.

In Figure 3, the filter in Table 2 identifies areas of low density related to cavity inside the propellant.

\section{CRITERIA OF ACCEPTANCE}

The criteria for the evaluation of discontinuities are the product of experience in the development of solid propellants. When a discontinuity is observed in the tomography image, first its location is determined and then its extension and the effects it would have on the combustion of the rocket engine are evaluated, this allows determining if the propellant is used in bench test, flight or is discarded.

- Location criteria in the plane (star periphery, star edge, others) : If the identified discontinuity is located in the periphery near the propellant inhibitor jacket, the observation could be raised since it would not affect the integrity of the propellant during its combustion. The discontinuity located in the valley of the star attached to the surface, in this case the firing speed of the propellant makes this discontinuity disappear quickly without affecting the combustion process, however, its shape, size and size must be considered in the discontinuity. location to determine the risk that could be involved. The discontinuity located on the crest (tip) of the star attached to the surface is of care, the shape, size and location must be analyzed because this area is critical and is the point of concentration of tension when the propellant is burning at rocket motor operating speed pressure. If the discontinuity occurs elsewhere in the propellant, its shape, size, and location should be observed to assess its risks. In a propellant, long cracks, both radial and longitudinal, should be avoided, which may cause breakage and alteration of the burning surface of the propellant grain. 
- Length through grain criterion

- If the discontinuity has a horizontal length in the propellant greater than $5 \mathrm{~mm}$, it is discarded.

- If the discontinuity has a radial length in the propellant cut greater than $5 \mathrm{~mm}$, it is discarded.

- Observation type criteria (cavity or dense element) : It must be taken into account that a cavity is always more significant and decisive when deciding whether a discontinuity is acceptable or not, according to its shape, location and size; It is a point of possible failure that can become a crack or crack due to the pressure that the propellant is subjected to in its operation. This does not remove the possibility that a discontinuity of higher density as some metallic element should be discarded, as a strange element that can alter some operating parameter of the propellant combustion, such as the burning speed.

\section{APPLICATION OF THE METHOD}

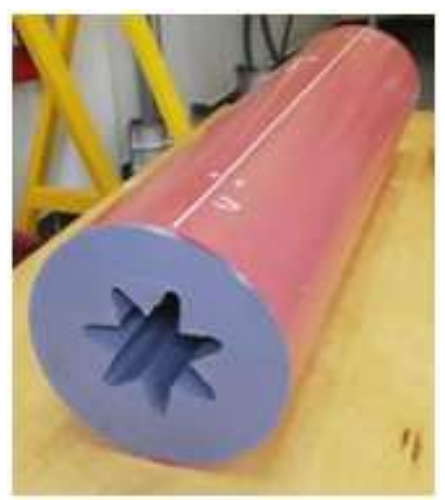

Figure 6 : PVC propellant

Figure 6.Shows the propellant before going through the tomography test, it has PVC as a binder, you can see the geometric configuration of the propellant and its red inhibitory jacket

\section{TOMOGRAPHY IMAGES MADE FOR THE PROPELLERS ELABORATED IN THE PERUVIAN} SPACE AGENCY - CONIDA YEAR 2019

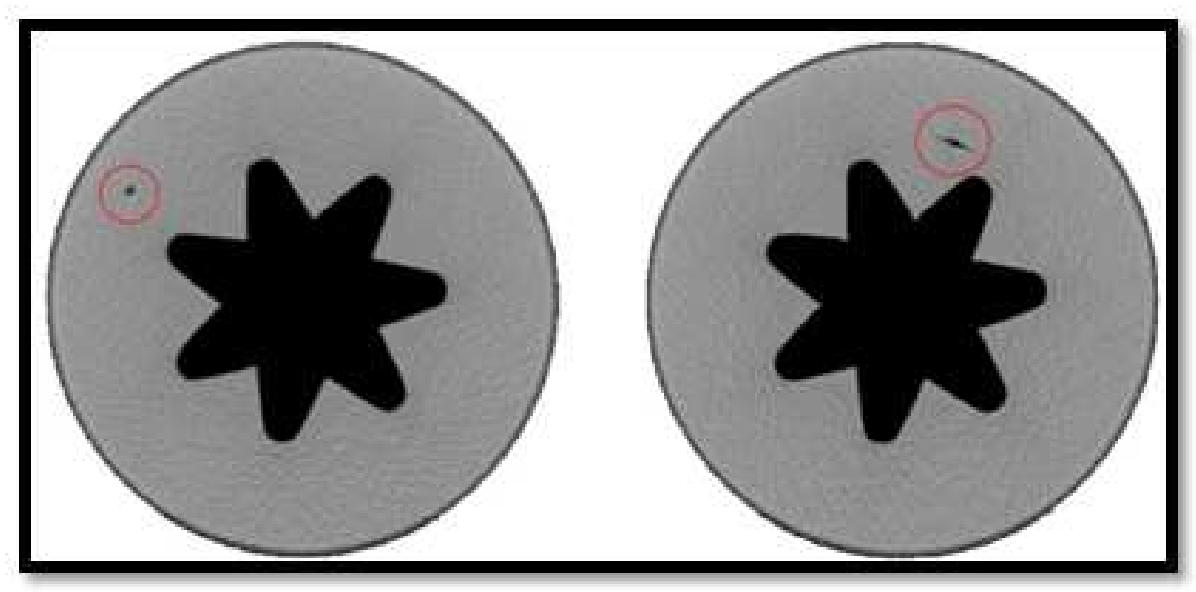

Figure 7 : Cut located $22 \mathrm{~cm}$ from nozzle section Figure 6. Cut located $34 \mathrm{~cm}$ from nozzle section 


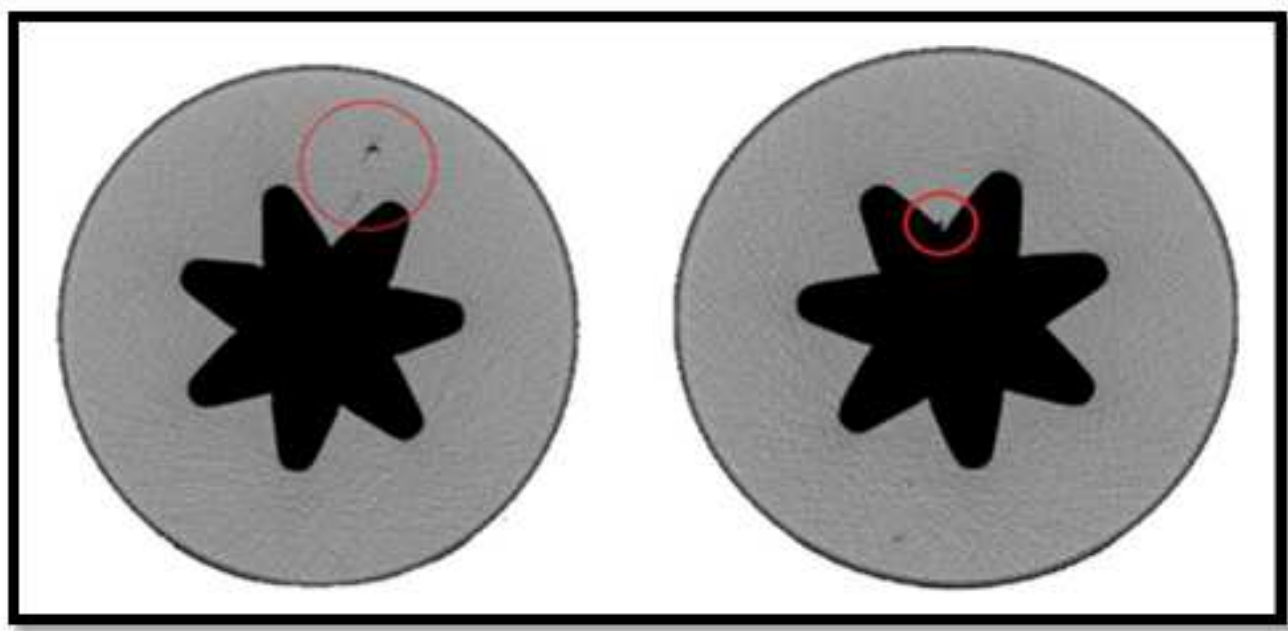

Figure 8 : Cut located at $71 \mathrm{~cm}$ of nozzle section and Cut located at $30.75 \mathrm{~cm}$ of nozzle section

Depth: Depth:

Bones: $5 \mathrm{~mm}$ Bones: $3.125 \mathrm{~mm}$

Brain base: $0.625 \mathrm{~mm}$ Brain base: $0.625 \mathrm{~m}$

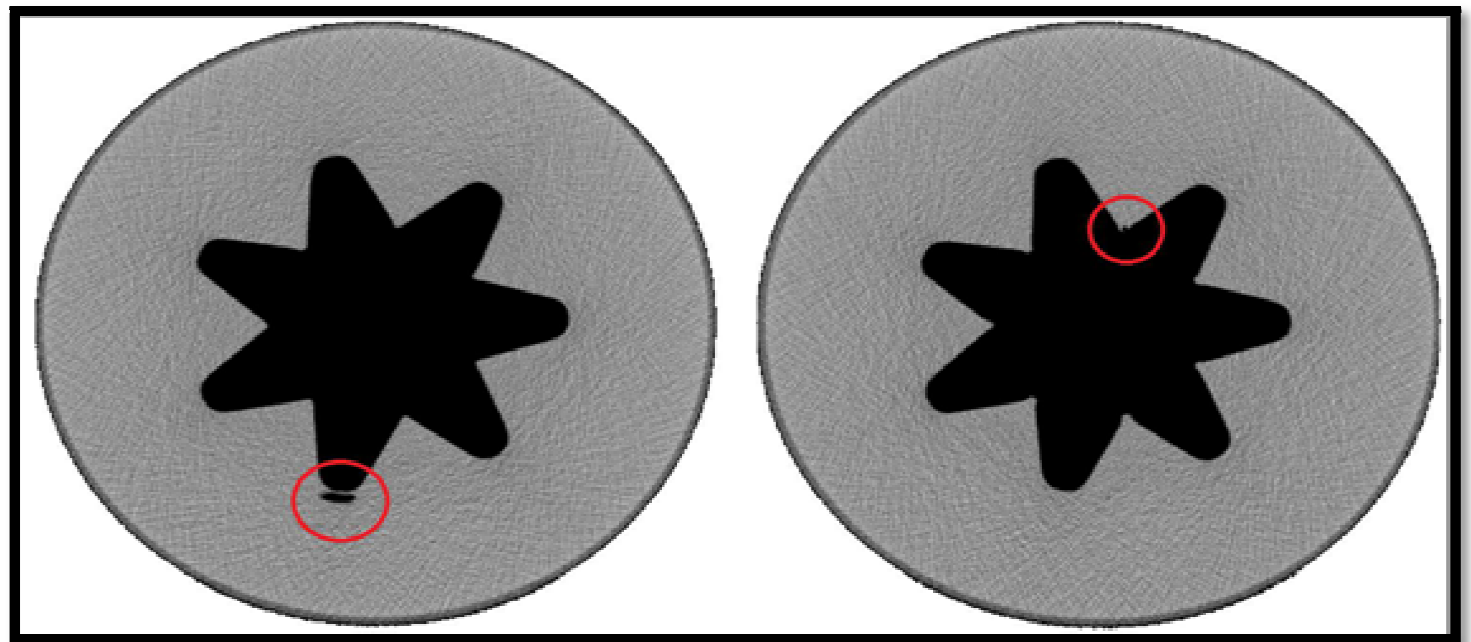

Figure 9 : Cut located at $2.31 \mathrm{~cm}$ of nozzle section Figure 10 . Cut located at $66.13 \mathrm{~cm}$ of nozzle section

Depth:

Bones: $3.75 \mathrm{~mm}$

Brain base: $3.75 \mathrm{~mm}$
Depth:

Bones: $2.5 \mathrm{~mm}$

Brain base: $1.25 \mathrm{~mm}$ 


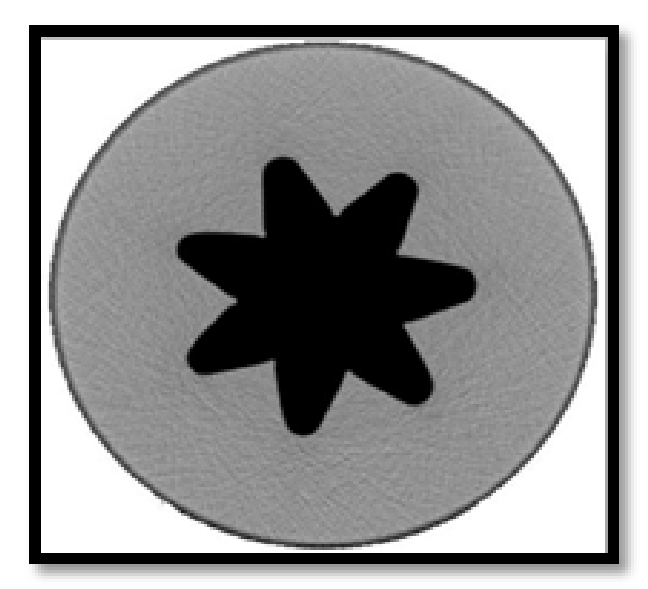

Figure 10 : Cut without blemishes

\section{RESULTS}

- The images of tomography in sections, have allowed to clearly identify the discontinuities in different shapes and sizes in the propellant.

- The images obtained using the filters of table 1 and 2 in the tomograph allow evaluating the entire internal surface of the propellant, where the imperfections that are generated during the development stage of the PVC propellant are observed.

- These results allow corrections to be made in the propellant manufacturing process and minimize the failures that may occur in the operation of a rocket motor.

- The cavities shown in fig. 5 is encapsulated and located at a distance of $220 \mathrm{~mm}$ from the nozzle section. Figures 6 and 7 present a cut surface that, during engine operation, can open and cause failure in the combustion chamber, while Figure 8 corresponds to a very small star tip anomaly. Figure 9 shows a cavity in the propellant valley, not superficially visible being $23.1 \mathrm{~mm}$ from its base, and Figure 10 shows a very insignificant star tip imperfection. In these cases the imperfections detected by the non-destructive computed tomography test would be acceptable.

- Figure 11 corresponds to the homogeneous image of the inner surface of a propellant without blemishes.

\section{CONCLUSIONS}

- The tomography applied to solid propellants is a fundamental tool because it allows to accurately show images in each cut.

- The real magnitudes and those presented by the tomographic image are very precise, and the thickness and dimensions of the observations found can be verified.

- The application of the non-destructive computed tomography test applied to solid propellants composed of PVC, provides relevant information through images of the internal surfaces which allows quality controls to be carried out according to the established acceptance criteria to qualify or rule out their use in engines. rocket.

- Guarantees the selection of the propellant for its quality and homogeneity to be used in a rocket engine

- It allows to improve the process parameters in the development of solid propellants. 


\section{REFERENCES}

1. Feature extraction from micro-tomography analysis of solid propellants, Filippo Maggi * and Marco Bratti $\dagger$ 8TH European Conference for Aeronautics and Aerospace Sciences (Eucass) DOI: 10.13009/EUCASS2019-706, pág.1

2. Krzysztof Tomkiewicz1, Andrzej Plaskowski2, Maurice S. Beck3, Malcolm Byars4, Testing of the Failure of the Solid Rocket Propellant with Tomography Methods, 1st World Congress on Industrial Process Tomography, Buxton, Greater Manchester, April 14-17, 1999. Page 1

3. Lamarque P., Tauzia J.M., Assessment of the ShelfLife of SRM: Prospects provided by High Energy X-Rays Computed Tomography, Paper No 15, AGARD/PEP Symposium "Service life of Solid propellant Systems ", Athens, May 1996.

4. Practice NO. PT-TE-1436 Advanced Computed X-Ray Tomography page 1

5. Ultrasonic Tomographic Imaging of an Encased, Highly Attenuating Solid Medium by T.H, Gan, D.A. hutchins, D.R Billson \& F.C. Wong Research in Nondestructive Evaluation volume 13, pages131-152(2001) Cite this article 